\title{
Runs of Homozygosity and Genomic Inbreeding Concepts in Dairy Animal Breeding
}

\author{
N. Anand Kumar ${ }^{1 *}$, C.G. Shashank ${ }^{2}$ and P.S. Banakar ${ }^{3}$ \\ ${ }^{1}$ Department of Animal Genetics and Breeding, ${ }^{2}$ Department of Animal Physiology, \\ ${ }^{3}$ Department of Animal Nutrition, ICAR-NDRI Karnal, Haryana-132001, India
}

*Corresponding author

\section{A B S T R A C T}

\section{Keywords \\ Runs of Homozygosity $(\mathrm{ROH})$, Genomic \\ Inbreeding, Inbreeding \\ Coefficient, Genome, SNP Chip \\ Article Info \\ Accepted: \\ 06 July 2018 \\ Available Online: \\ 10 August 2018}

Inbreeding among farm animals is common practice to maintain purity of a specific population and also is unavoidable due to use of few male animals compared to large number of females in any animal breeding farm. Level of inbreeding practiced in a farm is very crucial as the inbreeding depression causes loss of productivity and vitality of the breeding animals. Measuring inbreeding coefficient needs pedigree of each animal which is often not maintained in most of farms, or is incorrect and limited to a very few generations. Runs of homozygosity patterns in genome of individuals can capture inbreeding of most distant origin and gives a very accurate picture of level of inbreeding present in a farm even in absence of complete pedigree and also are helpful in evolutionary studies and selection signatures analysis.

\section{Introduction}

Inbreeding, the method of mating of genetically related individuals of a population as described by Wright, in 1922 is a vital tool in population genetic study of farm animal breeding researches as it effects the level of genetic variability present between groups of animals of a population and affect the production potential and fitness levels of inbred animals (Saccheri et al., 1996). Traditional method of calculating the extent of inbreeding in animals involves two methods. First method is recording pedigree of a particular individual up to a certain number of generations and then connecting the individual with its ancestors through the pathways of distant common ancestors known as pathway method (Wright, 1922). The second tedious method of estimating inbreeding coefficient is Covariance analysis, which consumes more time for a complicated pedigree. The information generated by covariance analysis can be used to quickly predict inbreeding values that will be produced from any possible mating between individuals in the pedigree.

Both the methods have a common and very important limitation in calculating the 
accurate value of inbreeding coefficient, i.e. the pedigree information of the individual. In most of the cases thorough information about the ancestors of an individual may not be available and the available information may be incorrect especially in records where pen mating systems are used and it is difficult to ascertain the male parent of an individual.

The latest technological revolution in the field of genome sequencing has resulted in faster and accurate sequencing of entire genome for reduced cost and efforts (Bovine Hapmap Consortium, 2009). These advances in the genomic sequencing has shifted the focus of animal breeders from phenotypic inbreeding coefficient calculation to genome based inbreeding calculation based on Runs of Homozygosity $(\mathrm{ROH})$ patterns analysis.

$\mathrm{ROH}$ patterns vary between individuals and offer tremendous information about an individual's ancestry, production potential and also evolutionary studies. This review is intended to focus on the importance of $\mathrm{ROH}$ as a tool in animal breeding studies.

\section{Runs of Homozygosity ( $\mathrm{ROH}$ ) patterns}

Availability of high density SNP chip information has led to detection of long stretches of autozygous regions in human genomes (Broman and Weber, 1999). Individual autozygosity can be measured using Runs of Homozygosity ( $\mathrm{ROH})$ (Gibson et al., 2006; McQuillan et al., 2008; Keller et al., 2011; Ferenčaković et al., 2013), which are contiguous homozygous fragments of DNA sequence without heterozygosity in the diploid state. Broman and Weber (1999) stated that $\mathrm{ROH}$ are caused by common ancestors, and due to recombination events interrupting long chromosome segments over time, extensive $\mathrm{ROH}$ segments are supposed to be autozygous stretches originated from recent common ancestors. Shorter $\mathrm{ROH}$ segments originate from distant ancestors or may involve some non-IBD stretches (Howrigan et al., 2011).

The initial studies on $\mathrm{ROH}$ regions were done by Gibson et al., (2006) in humans and by Sölkner et al., (2010) in cattle. ROH pattern analysis involves calculation of genomic inbreeding coefficient (FROH) by following formula

$f R O H=\Sigma\left(\frac{\text { LROH }}{\text { Lauto }}\right)$

In which, $\mathrm{L}_{\mathrm{ROH}}$ is the length of the autosome under $\mathrm{ROH}$ and Lauto is the total length of autosome. Thus $\mathrm{F}_{\mathrm{ROH}}$ being based on entire autosome gives a better and accurate value of inbreeding. Purifield et al., (2012) compared $\mathrm{F}_{\mathrm{ROH}}$ with pedigree based inbreeding coefficients (Fped) of 9 different cattle breeds, and concluded that pedigree based inbreeding values underestimated ancient relatedness that may exist among breeds. Ferencakovic et al., (2013) studied 4 breeds namely Brown Swiss, Norwegian red, Fleckveih, and Tyrol Grey for comparing pedigree based and $\mathrm{ROH}$ based inbreeding coefficients and reported higher levels of genomic inbreeding values based on $\mathrm{ROH}$ than that of pedigree based values.

A study of artificial selection on the $\mathrm{ROH}$ patterns in three contemporary groups of Holstein cattle by Kim et al., (2013) revealed that genes governing traits of economic important traits were found in regions covered by ROH. Further the results were validated by measuring integrated Haplotype Score (iHS) and found that results were consistent with that of $\mathrm{ROH}$ findings. Changes in patterns of $\mathrm{ROH}$ between the groups selected and unselected for milk production were observed on chromosomes 1, 2, 7, and 20, which all are proven Quantitative Trait Loci (QTL) for milk production. 
Curik et al., 2014 studied ROH patterns and listed the advantages of $\mathrm{ROH}$ analysis in evaluating inbreeding coefficient as it is easier to partition $F_{R O H}$ into values for individual chromosome or for even a particular segment of a chromosome. Second advantage being $\mathrm{FROH}$ is that the reference population is clear: it is based on the expectation that two related individuals, or two gametes uniting in an individual, will share identical chromosomal segments (haplotypes) of a certain length, assuming they are IBD. Bjelland et al., (2013) used three methods to evaluate genomic inbreeding from whole genome SNP date of 5853 animals and concluded that $\mathrm{F}_{\mathrm{ROH}}$ was better than $F_{\text {ped }}$ and $F_{\text {GRM }}$ as only $F_{R O H}$ was able to differentiate markers that were Identical by State (IBS) and Identical by Descent (IBD), which is important for evaluating the effects of inbreeding. Furthermore, $\mathrm{F}_{\mathrm{ROH}}$ has been previously shown to be the most correlated with homozygous mutation load and can also exploit the concept of a base population more effectively than the other measures of genomic inbreeding.

Kim et al., (2015) compared pedigree records of 966 Jersey animals with molecular data of $\mathrm{ROH}$ to compare effect of selection, effect of $\mathrm{ROH}$ on phenotypes and genomic inbreeding. In all the cases, inbreeding coefficient based on $\mathrm{ROH}$ were higher than pedigree estimates. Association of $\mathrm{ROH}$ with Daughter Pregnancy Rate (DPR) and Somatic Cell Score (SCS) revealed negative association between ROH and DPR on BTA 3, 7, 8, and 12 suggesting potential influence of local autozygosity on fertility. Similarly, association test of SCS resulted in the directional effect of $\mathrm{ROH}$ on the trait. $\mathrm{ROH}$ that were associated with SCS also affected increased $F_{P E D}$ on BTA 1, 3, 4, 5, 13, and 21, suggesting that elevated homozygosity could be involved in the susceptibility to mastitis. The genes identified in the regions that were significantly associated with DPR or SCS. One of the largest clusters from the analysis of DPR and SCS, genes affecting cell communication and sensory cognition (COL4A2, COL4A1, GJA2, GJB3, and GJB6) are located on BTA $3(\sim 40 \mathrm{Mb})$. Annotation of the regions that were associated with SCS revealed several interesting genes that may be involved in biological pathways like immune response, including CBLB (BTA 1) and NCK1 (BTA 1) that are involved in T cell receptor signaling pathway.

Purfield et al., (2017) studied distribution of $\mathrm{ROH}$ and selection signatures in 3191 sheep belonging to six commercial meat breeds. The $\mathrm{ROH}$ hotspot with the highest occurrences was located on autosome 2 and likely candidate genes within this region include MSTN, ITGAV, BINI and NUP35, all of which are involved in muscle differentiation. Several regions under putative selection were identified on autosomes 2, 4, 9 and 23, and plausible candidate genes within these regions included the fertility related genes $N T R K 2$, HECW2, STK17B and ITGB8.

Keller et al., (2011) studied SNP data as a function of population size to investigate the variation in inbreeding depression. Three methods in the study viz. theoretical, simulated genetic data, and real genetic data were compared to find the optimal estimate of inbreeding coefficient and to estimate the power to detect inbreeding depression. Results indicated that $\mathrm{F}$ estimated from runs of homozygosity (Froh), which reflects shared ancestry of genetic haplotypes, retains variation in even large populations (e.g., $\mathrm{SD}=$ $0.5 \%$ when $\mathrm{Ne}=10,000)$ and is likely to be the most powerful method of detecting inbreeding effects from among several alternative estimates of $\mathrm{F}$.

ROH patterns, being long stretches of homozygous fragments in genome as a result 
of inbreeding can accumulate many deleterious variants across generations which can be analyzed to predict occurrence of a particular disease or the effect of a particular variant in a population. Zhang et al., (2015) used tool like Variant Effect Predictor (VEP) to check the effect of variant in $\mathrm{ROH}$ patterns obtained across 4 breeds of cattle and classified them as deleterious and nondeleterious variants. There was a very strong positive correlation between the number of deleterious homozygotes and the genomic $\mathrm{ROH}$ proportion. Similarly, the number of homozygotes outside of ROH decreased with the genomic $\mathrm{ROH}$ proportion due to smaller non- $\mathrm{ROH}$ regions as $\mathrm{ROH}$ coverage increased and there was a weak negative correlation between deleterious homozygotes outside $\mathrm{ROH}$ and the genomic $\mathrm{ROH}$ proportions. This indicated that the increased deleterious homozygotes in $\mathrm{ROH}$ regions exceeded deleterious homozygote in non- $\mathrm{ROH}$ regions in cattle.

This review clearly elaborates that level of inbreeding $(F)$ estimated from $\mathrm{ROH}(F \mathrm{ROH})$ has several advantages as compared to pedigree estimates (FPED) as follows:

$F \mathrm{ROH}$ can predict the actual percentage of the genome that is autozygous more precisely as compared to FPED;

FROH can capture autozygosity arising from very distant common ancestors (e.g., 50+ generations ago);

$F \mathrm{ROH}$ can be estimated in any genotyped individual, even though pedigree information is not available;

$F \mathrm{ROH}$ offer the possibility to examine the distribution of autozygosity across the genome and to find specific locations in the genome with higher levels of autozygosity (e.g., by estimating $F \mathrm{ROH}$ separately for different chromosome; Keller et al., 2011; Ferenčaković et al., 2013).

Nothnagel et al., (2009) has reported regions of "ROH islands" or "ROH hot spots" in humans that are present in more than $50 \%$ of the individuals studied, which could theoretically be an indication of strong past selection in these populations. Additionally, Pemberton et al., (2012) have revealed that $\mathrm{ROH}$ patterns are influenced not only by the locations of recessive-disease loci, but also by population history and mating systems. The study of $\mathrm{ROH}$ in different populations can provide insight into how population history, genomic properties, and cultural habits could affect the observed islands of $\mathrm{ROH}$ in the human genome (Pemberton et al., 2012). In this sense genome-wide autozygosity can be used for recognition of recessive disease variants using homozygosity mapping, as well as for investigating the effects of genomewide homozygosity on important traits (McQuillan et al., 2008; Keller et al., 2011).

Thus Runs of Homozygosity (ROH) patterns which are actually the byproducts of whole genome sequencing can be of great value for animal breeders as they can reveal a lot about the population's ancestry, evolution, and also the level of actual inbreeding occurring in the herd can be calculated even in absence of proper pedigree of animals.

\section{References}

Bjelland, K. A. Weigel, N. Vukasinovic, and J. D. Nkrumah. Evaluation of inbreeding depression in Holstein cattle using whole-genome SNP markers and alternative measures of genomic inbreeding.

Bovine HapMap Consortium, Gibbs, R.A., Taylor, J.F., Van Tassell, C.P., Barendse, W., Eversole, K.A., Gill, C.A., Green, R.D., Hamernik, D.L., 
Kappes, S.M., Lien, S., Matukumalli, L.K., McEwan, J.C., Nazareth, L.V., Schnabel, R.D., Weinstock, G.M., Wheeler, D.A., Ajmone-Marsan, P., Boettcher, P.J., Caetano, A.R., Garcia, J.F., Hanotte, O., Mariani, P., Skow, L.C., Sonstegard, T.S., Williams, J.L., Diallo, B., Hailemariam, L., Martinez, M.L., Morris, C.A., et al., (2009). Genome-wide survey of SNP variation uncovers the genetic structure of cattle breeds. Science 324: 528-532.

Broman K.W., Weber J.L. (1999) Long homozygous chromosomal segments in reference families from the Centre d'E 'tude du Polymorphisme Humain. Am. J. Hum. Genet., 65, 1493-1500.

Curik I, Maja Ferenčaković, and Johann Sölkner, Inbreeding and Runs of homozygosity: A possible solution to an old problem, Livestock Science, http://dx.doi.org/10.1016/j.livsci.2014.0 5.034

Ferenčaković M., Hamzic, E. Gredler, B. T.R, Solberg, G: Klemetsdal, I. Curik and J. Sölkner Sölkner Sölkner. (2013) Estimates of autozygosity derived from runs of homozygosity: empirical evidence from selected cattle populations. J. Anim. Breed. Genet., 130, 286-293.

Gibson M., Newton E. and Collins, Andrew. (2006) Extended tracts of homozygosity in outbred human populations. Human Molecular Genetics., 15, 789-795.

Howrigan D.P., Simonson M.A., Keller M.C. (2011) Detecting autozygosity through runs of homozygosity: a comparison of three autozygosity detection algorithms. BMC Genomics., 12, 1471-2164.

Keller M.C., Visscher P.M., Goddard M.E. (2011) Quantifi- cation of inbreeding due to distant ancestors and its detection using dense single nucleotide polymorphism data. Genetics., 89, 237249.
Kim E-S, Cole JB, Huson H, Wiggans GR, Van Tassell CP, et al., (2013) Effect of Artificial Selection on Runs of Homozygosity in U.S. Holstein Cattle. PLoS ONE 8(11): e80813. doi:10.1371/journal.pone.0080813

Kim E-S, Sonstegard TS, Van Tassell CP, Wiggans G, and Rothschild MF (2015). The Relationship between Runs of Homozygosity and Inbreeding in Jersey Cattle under Selection. PLoS ONE 10(7): e0129967. doi:10.1371/journal.pone.0129967.

McQuillan R., Leutenegger A.L., AbdelRahman R., Franklin C.S., Pericic M., Barac-Lauc L., Smolej-Naran- cic N., Janicijevic B., Polasek O., Tenesa A., Macleod A.K., Farrington S.M., Rudan P., Hayward C., Vitart V., Rudan I., Wild S.H., Dunlop M.G., Wright A.F., Camp- bell H., Wilson J.F. (2008) Runs of homozygosity in European populations. Am. J. Hum. Genet., 83, 359-372.

Nothnagel M., T. Lu, M. Kayser, M. Krawczak. (2009). Genomic and geographic distribution of SNP-defined runs of homozygosity in Europeans. Human Molecular Genetics., 19, 2927 . 2935.

Pemberton J.T., Devin Absher, Marcus W. Feldman, Richard M. Myers, Noah A. Rosenberg and Jun Z. Li. (2012) Genomic Patterns of Homozygosity in Worldwide Human Populations. Am. J. Hum. Genet, 91, 275-292.

Purfield DC, McParland S, Wall E, and Berry DP (2017). The distribution of runs of homozygosity and selection signatures in six commercial meat sheep breeds. PLoS ONE 12(5): e0176780. https://doi.org/10.1371/journal.pone.017 6780.

Saccheri I.J., Brakefield P.M., Nichols R.A. (1996). Severe inbreeding depression and rapid fitness rebound in the 
butterfly Bicyclusanynana (Satyridae) Evolution. 50:2000-2013.

Sölkner. (2010) Runs of Homozygosity Reveal Genome-wide Autozygosity in the Austrian Fleckvieh Cattle. Agriculturae Conspectus Scientificus., $76,325-328$.
Wright S. Coefficients of inbreeding and relationship. Amer Naturalist 1922, 56:330-338.

Zhang, Bernt Guldbrandtsen, Mirte Bosse, Mogens, Lund and Goutam Sahana. Runs of homozygosity and distribution of functional variants in the cattle genome. BMC Genomics (2015) 16: 542. DOI 10.1186/s12864-015-1715-x

\section{How to cite this article:}

Anand Kumar, N., C.G. Shashank and Banakar, P.S. 2018. Runs of Homozygosity and Genomic Inbreeding Concepts in Dairy Animal Breeding. Int.J.Curr.Microbiol.App.Sci. 7(08): 688-693. doi: https://doi.org/10.20546/ijcmas.2018.708.075 